\title{
GANCICLOVIR-RESISTANT CYTOMEGALOVIRUS IN PAEDIATRIC PATIENTS
}

\section{Sophida Boonsathorn, Nopporn Apiwattanakul, Chonnamet Techasaensiri}

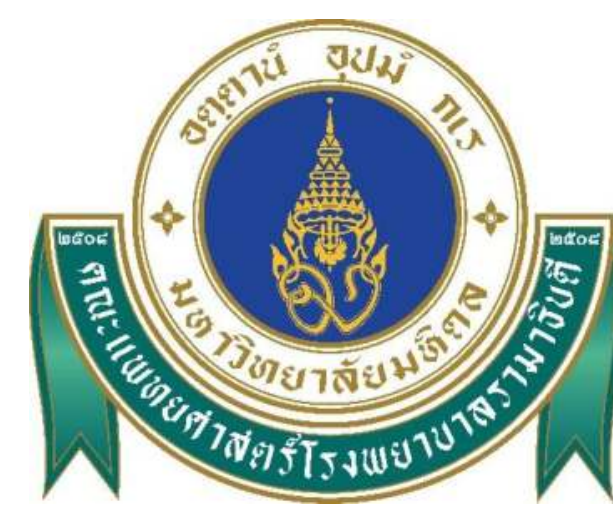

Division of Paediatric Infectious Diseases, Department of Paediatrics, Faculty of Medicine Ramathibodi Hospital, Mahidol University, Bangkok, Thailand

\section{Background}

Cytomegalovirus (CMV) infection is one of the leading causes of morbidity and mortality among haematopoietic stem cell and solid organ transplant patients. Recently, the emergence of CMV-drug resistance has been recognised among those who were treated with ganciclovir. However, there is still limited data in paediatric patients. We aimed to describe clinical characteristics and outcomes of CMV infection with ganciclovir resistance in paediatric patients.

\section{Methods}

We retrospectively identified 10 patients from 2013 to 2017 who had genotypically confirmed ganciclovirresistant CMV infection. Genotypic assays for UL97 gene mutations were analysed by RT-PCR. Demographic data, clinical manifestations, virological data, treatment, and outcomes were collected from electronic records.

\section{Results}

The median age of the patients was 3 years (interquartile range [IQR], 0.84-8.67). Four transplanted recipients (3 liver, 1 kidney) and six oncologic patients (5 leukemia, 1 Infection-Associated Haemophagocytic Syndrome; IAHS) were included.

Ganciclovir resistance was tested at a median time of 22.5 days (IQR, 14.3-31) after initiation of treatment with ganciclovir with median CMV load of 41,100 copies/mL (IQR, 13,575-100,982) or log 4.61 (IQR, 4.13-5.0). All ganciclovirresistant isolates harboured a UL97 mutation in codon 460 . A median of maximum CMV load was 113,245 copies/mL (IQR, 54,900-1,184,635) or log 5.05 (IQR, 4.74-6.05).

None of our patients had received ganciclovir or valganciclovir for CMV prophylaxis. Six of 10 patients $(60 \%)$ were successfully treated with high-dose ganciclovir (7.5 mg/kg twice daily). Other received foscanet and cidofovir. Four patients died due to non-CMV-related problems.

\section{Conclusion}

Ganciclovir-resistant CMV infection is an emerging problem, not only in transplanted recipients but also oncologic patients. Therefore, screening for mutations should be considered in patients with persistent viremia, even though patients had not received ganciclovir prophylaxis before and treatment with high-dose ganciclovir could be an effective treatment modality

Table: Demographic and Clinical Characteristics of 10 Patients With Drug-Resistant CMV Infection

\begin{tabular}{|l|c|}
\hline \multicolumn{1}{|c|}{ Characteristic } & (n = 10) \\
\hline Male sex (n, \%) & $5(50)$ \\
\hline $\begin{array}{l}\text { Median age at CMV diagnosis, } \\
\text { years (IQR) }\end{array}$ & $3.0(0.84-8.67)$ \\
\hline $\begin{array}{l}\text { Underlying disease (n, \%) } \\
-\quad \text { Solid-organ transplantation } \\
-\quad \text { Hematologic malignancy }\end{array}$ & $4(40)$ \\
\hline $\begin{array}{l}\text { CMV manifestation (n, \%) } \\
-\quad \text { Tissue-invasive }\end{array}$ & $5(60)$ \\
\hline $\begin{array}{l}\text { Median days prior to ganciclovir- } \\
\text { resistant diagnosis, days (IQR) }\end{array}$ & $22.5(13.5-31)$ \\
\hline $\begin{array}{l}\text { Median viral load at ganciclovir- } \\
\text { resistant was suspected (IQR), log }\end{array}$ & $4.67(4.26-5.04)$ \\
\hline Median peak viral load (IQR), log & $5.09(4.86-6.13)$ \\
\hline Death (n, \%) & $4(40)$ \\
\hline
\end{tabular}

